\title{
REPLICA-SYMMETRY BROKEN SOLUTION OF SIMPLE PSEUDOSPIN MODEL OF ORIENTATIONAL GLASS
}

\author{
K. Walasek, K. Lukierska-Walasek and M. Wodawski \\ Institute of Physics, Pedagogical University of Zielona Góra \\ Plac Slowiański 6, 65-069 Zielona Góra, Poland
}

The first stage of the replica-symmetry breaking for a simple quadrupolar glass model using the Parisi ansatz has been performed in a wide range of temperatures including $T=0$. It has been obtained that the entropy of the system increases considerably compared to that calculated within the replica symmetric theory.

PACS numbers: $64.60 . \mathrm{Cn}, 64.70 . \mathrm{PC}, 62.20 . \mathrm{Dc}, 75.10 . \mathrm{Nr}$

Much effort has been dedicated in recent years to the clarification of the nature of non-magnetic frustrated systems such as Potts and quadrupolar glasses (QGs) [1]. Though these systems have been considered mostly within the replica symmetric (RS) theory some attempts to study the replica-symmetry breaking (RSB) mechanism have been made [1-3]. However, despite the success of the Parisi RSB scheme [3] for magnetic systems with the spin reversal symmetry (the Ising and $m$-vector model) a convincing theory does not exist for richer models of disordered systems. The analysis of the RSB problem for quadrupolar and Potts glasses has been performed using the simplified Landau free energy of the system expanded up to fourth order into the glassy order parameter. Such an approach is limited to the range where the order parameter is sufficiently small, but it is not adequate for the study of the system at lower temperatures. In general, the study of properties of such systems in the whole range of temperature (without Landau expansion) is rather a complicated task because of the complexity of interactions which makes it difficult to express the free energy in a closed form. Nevertheless, it would be interesting and desirable to attempt to perform RSB investigations for a possible simplest model. We have in mind the quadrupolar system with a strong anisotropy in the $z$ direction described by the following $S=1$ spin (or pseudospin) Hamiltonian:

$$
H=\sum_{i, j} J_{i j} \mathcal{O}_{i}^{0} \mathcal{O}_{j}^{0}
$$

where $J_{i j}$ denotes the coupling between quadrupoles located at sites $i, j$ and

$$
\mathcal{O}_{i}^{0}=3\left(S_{i}^{z}\right)^{2}-2 \text {. }
$$


It is assumed that $J_{i j}$ 's are quenched random interactions of infinite range independently distributed according to the Gaussian probability distribution with zero mean and variance $J / \sqrt{N}$, where $N \rightarrow \infty$ denotes the number of spins (pseudospins).

As it was discussed in Ref. [4] some limited insight into the nature of the quadrupolar glassy freezing of randomly distributed molecules of $o-\mathrm{IH}_{2}$ in a matrix of spherical parahydrogen species can be obtained from the model (1). In this case $S=1$ denotes the rotational quantum number of quadrupole-bearing molecule of $0-\mathrm{II}_{2}$.

The model (1) has been solved within the RS theory [5, 6]. In Ref. [7] the stability limit of the RS phase of the model (1) has been studied. It has been found that above certain temperature $T_{\mathrm{c}}=1.367 \mathrm{~J} / k_{\mathrm{B}}$ the RS phase is stable, whereas at $T<T_{\mathrm{c}}$ becomes unstable. This means that as the temperature is lowered and reaches $T_{\mathrm{c}}$ the system undergoes a transition from ergodic to nonergodic phase with multiple minima of free energy characteristic of the glassy state with the broken replica symmetry. However, this is not a phase transition in an usual thermodynamic sense since the QG order parameter increases continuously with the decreasing of temperature $[5,6]$. Such a situation is observed in solid ortho-parahydrogen mixtures [4]. The order parameter is not a small quantity near $T_{\mathrm{c}}$. Hence in order to study our system we cannot use (except maybe of the region of very high temperatures) the Landau expansion for the free energy. According to the knowledge of the authors, existing investigations about RSB in QGs have used the Landau expansion $[1,2]$.

The aim of the present paper is to study the RSB theory for the model (1) in the wide region of temperature, that is for $0 \leq T \leq T_{\mathbf{c}}$, using the Parisi ansatz [3]. We confine ourselves to the first stage of $\mathrm{RSB}$ expecting that even the simplest approach will give us some insight into the RSB mechanism in QGs.

On proceeding in the strict analogy to the first stage of RSB scheme in Ref. [3] we get the free energy in the form

$$
\begin{aligned}
\frac{F}{N}= & \frac{\beta J^{2} \bar{x}}{4}\left(q_{1}^{2}-q_{0}^{2}\right)-\frac{\beta J^{2}}{4}\left(q_{1}+m-2\right)\left(q_{1}-m-2\right) \\
& -\frac{1}{\beta \bar{x}} \int_{-\infty}^{\infty} \mathrm{d} P_{q_{0}}(y) \ln \int_{-\infty}^{\infty} \mathrm{d} P_{q_{1}-q_{0}}(z) Z_{0}^{\bar{x}}
\end{aligned}
$$

where $q_{0}$ and $q_{1}$ denote two QG order parameters (characteristic of the first stage of RSB), $m$ is the quadrupolar long ranged order parameter assumed to be the same for each replica, $\bar{x}$ is understood as a real number in the interval between 0 and 1 ,

and

$$
\mathrm{d} P_{a}(\xi)=\frac{1}{\sqrt{2 \pi a}} \exp \left(-\frac{\xi^{2}}{2 a}\right) \mathrm{d} \xi,
$$

$$
Z_{0}=2 \mathrm{e}^{\vartheta}+\mathrm{e}^{-2 \vartheta}
$$

with

$$
\vartheta=\beta J(y+z)-\frac{(\beta J)^{2}}{2}\left(2-m-q_{1}\right) .
$$


The parameters $q_{1}, q_{0}, m$, and $\bar{x}$ are calculated from stationary conditions for $F$, i.e.

$$
\frac{\partial F}{\partial q_{1}}=0, \quad \frac{\partial F}{\partial q_{0}}=0, \quad \frac{\partial F}{\partial m}=0, \quad \frac{\partial F}{\partial \bar{x}}=0 .
$$

To study the effect of the first stage RSB on the thermodynamic properties of the system we calculate the entropy

$$
\frac{S}{N}=-\frac{\partial}{\partial T}\left(\frac{F}{k_{\mathrm{B}} N}\right)
$$

with $F / N$ given by Eq. (3).
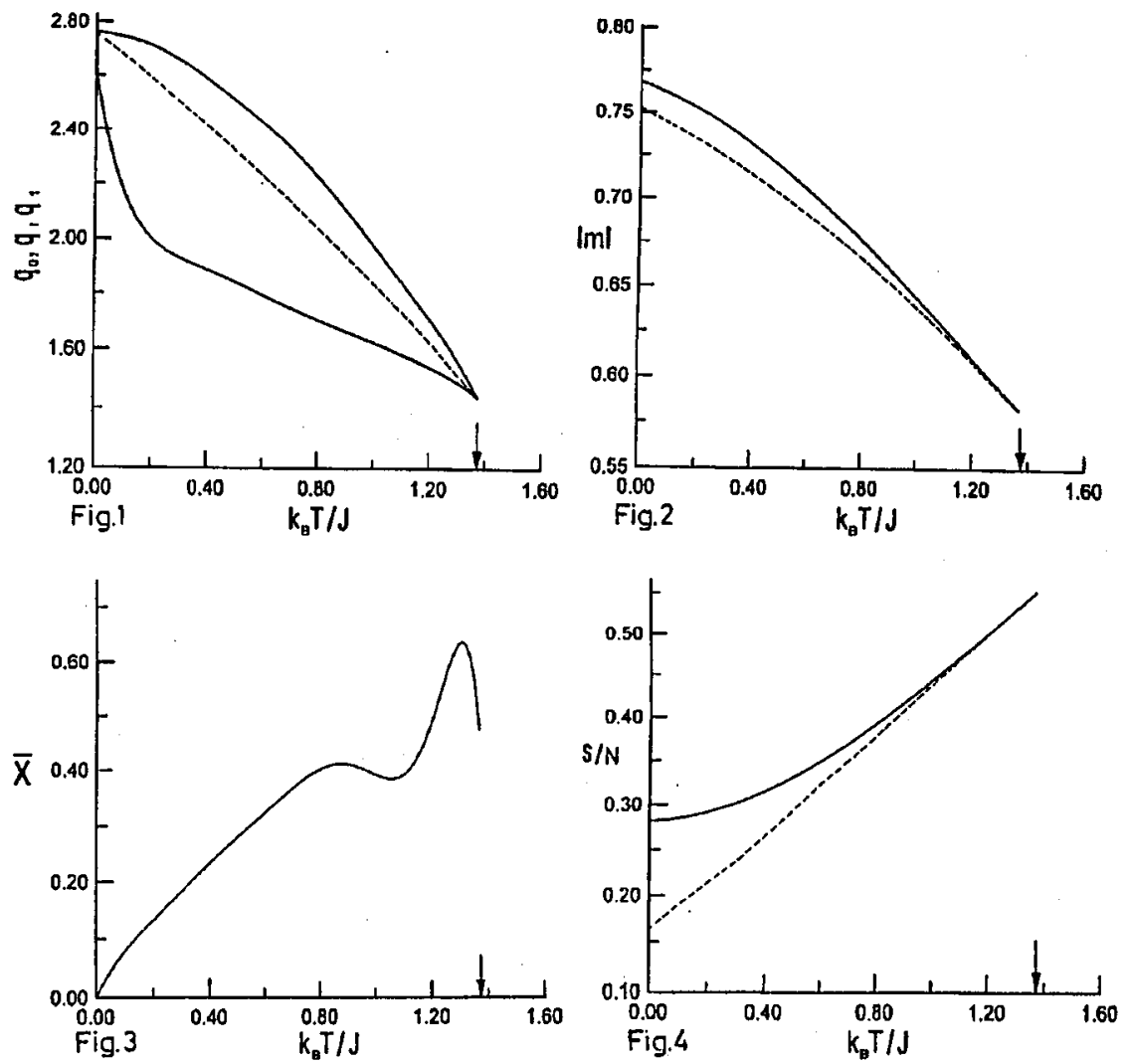

Fig. 1. The temperature dependence of the quadrupolar glass order parameters: $q_{1}$ upper solid line, go - lower solid line, and $q$ (replica symmetric theory) - dashed line. Fig. 2. The $|m|$ as a function of temperature. The first stage of replica symmetry breaking (solid line) and repl'sa-symmetry theory (dashed line).

Fig. 3. A variation of $\bar{x}$ with temperature.

Fig. 4. The temperature dependence of the entropy $S$. The solid and dashed lines refer to the replica-symmetry broken and replica-symmetry solutions, respectively. 
In order to obtain the temperature dependence ( $T$ dependence) of the parameters $q_{1}, q_{0}, m$ and $\bar{x}$ we have solved numerically Eqs. (7) for $0 \leq T \leq T_{\mathrm{c}}$. A variation of QG parameters $q_{1}$ and $q_{0}$ with $T$ is presented in Fig. 1. For a comparison the $T$ dependence of the QG parameter obtained within the RS theory $[5,6]$ is also given in this figure. In Fig. 2 the absolute value of the quadrupolarization (the quadrupolarization is always negative) as a function of the temperature is shown for the RSB as well as for RS theory [5,6]. Figure 3 contains the $T$-dependence of $\bar{x}$. It is seen that $\bar{x}$ has physical non negative values but as $T$ approaches $T_{\mathrm{c}}$ this variable slightly oscillates. Finally in Fig. 4 the entropies calculated within the RSB and RS theories are given. It is seen that the first stage of RSB leads to the considerable increasing of entropy, particularly at low temperatures, compared to the RS result. This means that RSB solutions are closer to a physical reality than the ones obtained within the RS scheme $[5,6]$.

\section{References}

[1] K. Binder, J.D. Reger, Adv. Phys. 41, 547 (1992) and references therein.

[2] P.M. Goldbart, Ph.D. Thesis, University of London, London 1985.

[3] M. Mezard, G. Parisi, M.A. Virasoro, Spin Glass Theory and beyond, in series World Scientific Lecture Notes in Physics, Vol. 9, World Scientific, Singapore 1987.

[4] N.S. Sullivan, Can. J. Chem. 66, 908 (1988).

[5] E.A. Lutchinskaia, V.N. Ryzhov, E.E. Tareyeva, J. Phys. C 17, L665 (1984).

[6] E.A. Lutchinskaia, V.N. Ryzhov, E.E. Tareyeva, Teor. Mat. Fiz. 67, 463 (1986) (in Russian).

[7] K. Walasek, Phys. Rev. B 51, 9314 (1995). 